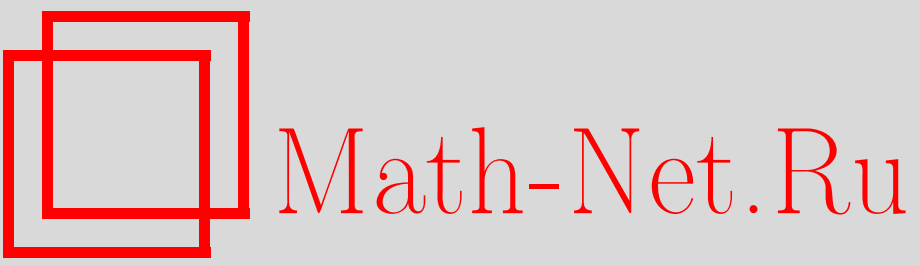

А. В. Гласко, Об одном свойстве оператора ренормализационной группы, ТMФ, 2004, том 138, номер 1, 71-80

DOI: https://doi.org/10.4213/tmf9

Использование Общероссийского математического портала Math-Net.Ru подразумевает, что вы прочитали и согласны с пользовательским соглашением

http://www.mathnet.ru/rus/agreement

Параметры загрузки:

IP : 52.90 .164 .192

26 апреля 2023 г., $17: 40: 20$ 
ТЕОРЕТИЧЕСКАЯ

И МАТЕМАТИЧЕСКАЯ

ФИЗИКА

Том 138, № 1

январь, 2004

(C) 2004 г.

А.В. Гласко*

\section{ОБ ОДНОМ СВОЙСТВЕ ОПЕРАТОРА РЕНОРМАЛИЗАЦИОННОЙ ГРУППЫ}

На примере изотропного ферромагнетика показано, что оператор ренормализационной группы можно рассматривать как оператор эволюции системы спинов с ростом приведенной температуры, т.е. как упорядочиваюший оператор системы.

Ключевые слова: ренормализационная группа, преобразование Каданова, критическая точка, ферромагнетик, оператор эволюции, упорядочивающий оператор.

\section{1. ВВЕДЕНИЕ}

Хорошо известно, что ренормализационная группа (РГ) играет важную роль в теоретической физике [1]-[4]. В то же время до сих пор обсуждаются два принципиальных вопроса [3]: о связи понятий РГ в квантовой теории поля и в теории критических явлений и о физическом смысле РГ.

В настоящей работе предлагается возможная физическая интерпретация РГ в теории критических явлений.

В этой теории оператор РГ $\widehat{R}_{s}$ рассматривается как некоторое преобразование симметрии для гамильтониана системы многих частиц [5], не имеющее непосредственного физического смысла, т.е. преобразование внутри множества моделей системы (от одного модельного гамильтониана к другому) [1], [3]. Связь этого преобразования с поведе-

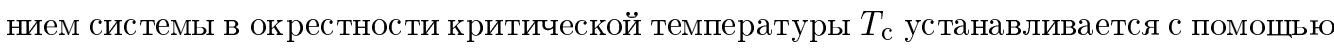
известной асимптотической формулы [5], [6]

$$
\widehat{R}_{s} \mu(\tau) \approx \mu^{*}+A \tau s^{y_{1}} \mathbf{e}_{1}+f(s), \quad s \rightarrow \infty
$$

где $\tau=1-T / T_{\mathrm{c}}$ - приведенная температура, $\mu$ - точка в пространстве параметров гамильтониана, представляюшая состояние системы, $\mu^{*}-$ неподвижная точка $\left(\widehat{R}_{s} \mu^{*}=\right.$ $\left.\mu^{*}\right), s^{y_{1}}$ - собственное значение линеаризованного оператора РГ, $\mathbf{e}_{1}$ - соответствующий собственньй вектор, $A$ - некоторая постоянная, $f(s)$ - бесконечно малая величина.

\footnotetext{
* Московский государственный технический университет, Москва, Россия
} 
Эта формула выражает ряд предположений о свойствах плотности распределения системы и позволяет получить все законы критического поведения физических величин (в отсутствие магнитного поля) и законы подобия.

В этой работе РГ будет исследоваться на примере модели изотропного ферромагнетика (размерность пространства $d=3$, число компонент параметра порядка $n=3$ ). Будет показано, что если рассматривать ферромагнетик просто как систему независимо распределенных магнитных моментов ("классических спинов"), равных по модулю единице, то оператор РГ в пределе малых $\tau$ можно отождествить с оператором эволюции плотности вероятности этой системы с ростом $\tau$, т.е. упорядочивающим оператором системы спинов. Более того, формула (1), по-сушеству, и выражает условие, чтобы оператор РГ был эволюционным оператором плотности. Таким образом, преобразование РГ трактуется уже не как преобразование внутри множества моделей, а как переход между физическими состояниями системы при разных значениях температуры.

\section{2. МОДЕЛЬ}

Будем рассматривать ферромагнетик как систему ячеечных спинов, причем последние определим как классические (неквантовые) векторы (магнитные моменты), по модулю равные единице. В качестве вектора состояния этой системы примем плотность вероятности $p(\boldsymbol{\sigma})$ того, что случайно выбранный спин системы равен $\boldsymbol{\sigma}$. Таким образом, будем описывать множество ячеечных спинов как совокупность независимо распределенных по закону $p(\boldsymbol{\sigma})$ случайных величин. Пространственное распределение ориентаций спинов нас интересовать не будет (ср. с [7]).

Как известно, при температуре ниже точки Кюри $(\tau>0)$ ферромагнетик обладает отличной от нуля намагниченностью $\mathbf{m}$. При уменьшении температуры (с ростом $\tau$ ) эта намагниченность монотонно растет по модулю, не меняясь по направлению, и достигает максимального значения при абсолютном нуле $(\tau=1)$. Примем направление вектора намагниченности $\mathbf{m}$ за направление оси $z$ и будем описывать систему спинов в сферической системе координат. Найдем обший вид плотности вероятности $p(\boldsymbol{\sigma})$ при $0 \leqslant \tau \leqslant 1$. Так как все спины по модулю равны единице $(|\boldsymbol{\sigma}|=\sigma=1)$, то

$$
p(\sigma)=\delta(\sigma-1) f(\varphi, \theta),
$$

где $\theta$ - угол, составляемый спином $\boldsymbol{\sigma}$ с осью $z, \varphi$ - полярньй угол ориентации спина.

Понятно, что распределение спинов по направлениям аксиально-симметрично относительно оси $z$, поэтому

$$
p(\boldsymbol{\sigma})=\frac{1}{2 \pi} \delta(\sigma-1) y\left(\sigma_{z}\right),
$$

где $\sigma_{z}$-проекция спина на ось $z, \sigma_{z}=\cos \theta$. Плотность $y\left(\sigma_{z}\right)$ является функцией приведенной температуры.

Рассмотрим эволюцию системы спинов с ростом $\tau$. При $\tau=0$ спины ориентированы абсолютно хаотически:

$$
y\left(\sigma_{z}\right)=y_{0}=\frac{1}{2},
$$


т.е. вероятность того, что произвольно выбранный спин имеет любую проекцию на ось $z$, одинакова. При этом

$$
p(\boldsymbol{\sigma})=p_{0}(\boldsymbol{\sigma})=\frac{1}{4 \pi} \delta(\sigma-1)
$$

При $\tau=1$ система спинов абсолютно упорядочена:

$$
y\left(\sigma_{z}\right)=\delta\left(\sigma_{z}-1\right)
$$

т.е. все спины ориентированы в направлении оси $z$. При этом

$$
p(\boldsymbol{\sigma})=\frac{1}{2 \pi} \delta(\sigma-1) \delta\left(\sigma_{z}-1\right)
$$

С ростом $\tau$ от 0 до 1 мера упорядоченности системы [8] растет и система эволюционирует из состояния абсолютного хаоса в состояние абсолютного порядка,

$$
p(\sigma, \tau)=\frac{1}{2 \pi} \delta(\sigma-1) y\left(\sigma_{z}, \tau\right) .
$$

\section{3. ЭВОЛЮЦИОННЫЙ ОПЕРАТОР}

Обозначим через $\widehat{\mathcal{E}}_{\tau}$ эволюционный оператор плотности вероятности, определенный соотношением

$$
\widehat{\mathcal{E}}_{\tau} p(\tau, \boldsymbol{\sigma})=\frac{1}{2 \pi} \delta(\sigma-1) \delta\left(\sigma_{z}-1\right), \quad \tau>0
$$

т.е. оператор, переводящий состояние системы в "момент" $\tau$ в конечное (абсолютно упорядоченное) состояние.

Переходя в равенстве (2) к пределу $\tau \rightarrow 0$, получим

$$
\widehat{\mathcal{E}}_{0} p_{0}=\lim _{\tau \rightarrow 0} \widehat{\mathcal{E}}_{\tau} p(\tau, \boldsymbol{\sigma})=\frac{1}{2 \pi} \delta(\sigma-1) \delta\left(\sigma_{z}-1\right) .
$$

Эволюционньй оператор $\widehat{\mathcal{E}}_{0}$ переводит начальное состояние системы, т.е. абсолютньй хаос, в конечное, т.е. абсолютный порядок. Другими словами, это оператор, упорядочивающий систему спинов.

\section{4. ПРЕОБРАЗОВАНИЕ КАДАНОВА}

Первым этапом при построении РГ в теории критических явлений служит преобразование Каданова $\widehat{K}_{s}[5]$, обычно определяемое следуюшим образом. Разобьем ферромагнитный образец на кубические блоки, каждый из которых содержит $s^{3}$ кристаллических ячеек. Каждому такому (кадановскому) блоку припишем спин $\boldsymbol{\sigma}^{\prime}$, равный среднему значению спинов входяших в него ячеек $\boldsymbol{\sigma}_{i}$,

$$
\boldsymbol{\sigma}^{\prime}=\frac{1}{s^{3}} \sum_{i=1}^{s^{3}} \boldsymbol{\sigma}_{i}
$$


Гамильтониан полученной системы блочных спинов назьвается блочным гамильтонианом. Под преобразованием Каданова обычно понимается преобразование исходного (ячеечного) гамильтониана в блочный. Понятно, что это преобразование можно применять повторно (и неоднократно) уже к блочному гамильтониану.

Мы будем рассматривать оператор Каданова непосредственно как преобразование вектора состояния системы $p(\boldsymbol{\sigma})$, а не гамильтониана (функцией которого вектор состояния является),

$$
p^{\prime}\left(\boldsymbol{\sigma}^{\prime}\right)=\widehat{K}_{s} p(\boldsymbol{\sigma})
$$

Рассмотрим состояние системы спинов $p(\tau, \boldsymbol{\sigma})$ в малой правосторонней окрестности критической точки и определим, как преобразуется состояние этой системы под действием оператора Каданова при очень больших $s$, т.е. чему равен предел

$$
\lim _{s \rightarrow \infty} \widehat{K}_{s} p(\tau, \boldsymbol{\sigma})
$$

Очевидно, что при $s \rightarrow \infty$, т.е. когда размер кадановского блока неограниченно растет, блочные спины стремятся к среднему значению спина по системе, т.е. к намагниченности образца $\mathbf{m}(\tau)$,

$$
\lim _{s \rightarrow \infty} \sigma^{\prime}(s)=\mathbf{m}(\tau) .
$$

При малых положительных значениях $\tau$ намагниченность отлична от нуля и представляет собой вектор, направленньй вдоль оси $z$, с модулем, зависящим от $\tau$,

$$
\mathbf{m}=m(\tau) \mathbf{k}
$$

где $\mathbf{k}$ - орт оси $z$. Более конкретно, функция $m(\tau)$ является монотонно возрастаюшей функцией $\tau$, причем

$$
m(0)=0, \quad m(1)=1 .
$$

Из соотношений (5) и (6) ясно, что блочные спины

$$
\boldsymbol{\sigma}^{\prime}(s) \rightarrow m(\tau) \mathbf{k} \quad \text { при } \quad s \rightarrow \infty .
$$

Таким образом,

$$
\lim _{s \rightarrow \infty} \widehat{K}_{s} p(\tau, \boldsymbol{\sigma})=\delta\left(\boldsymbol{\sigma}^{\prime}-m(\tau) \mathbf{k}\right)=\frac{1}{2 \pi} \delta\left(\sigma^{\prime}-m(\tau)\right) \delta\left(\sigma_{z}^{\prime}-\sigma^{\prime}\right), \quad \tau>0 .
$$

Доказательство формулы (8) проведем на основании теоремы Чебышева. Случайные величины $\boldsymbol{\sigma}_{i}$ распределены независимо по одному закону $p(\boldsymbol{\sigma})$. Математическое ожидание величин $\sigma_{i}$ равно среднему значению спина по системе, т.е. намагниченности,

$$
\left\langle\boldsymbol{\sigma}_{i}\right\rangle=\mathbf{m}(\tau)
$$


Дисперсия величин $\boldsymbol{\sigma}_{i}$ конечна,

$$
D \boldsymbol{\sigma}_{i}=1-m^{2}(\tau)<\infty
$$

Действительно,

$$
D \boldsymbol{\sigma}_{i}=\left\langle\left(\boldsymbol{\sigma}_{i}-\left\langle\boldsymbol{\sigma}_{i}\right\rangle\right)^{2}\right\rangle=\left\langle\boldsymbol{\sigma}_{i}^{2}\right\rangle-\left\langle\boldsymbol{\sigma}_{i}\right\rangle^{2}
$$

Учитывая, что $\sigma_{i}^{2}=\sigma^{2}=1$, и принимая во внимание равенство (9), получим отсюда (10). На основании известного следствия теоремы Чебышева [9] заключаем, что последовательность случайных величин $\boldsymbol{\sigma}^{\prime}(s)(4)$ сходится по вероятности к $\left\langle\boldsymbol{\sigma}_{i}\right\rangle$, т.е.

$$
\boldsymbol{\sigma}^{\prime}(s) \stackrel{\mathrm{p}}{\longrightarrow} \mathbf{m} \quad \text { при } \quad s \rightarrow \infty,
$$

т.е. для любого числа $\varepsilon>0$ вероятность

$$
P\left(\left|\boldsymbol{\sigma}^{\prime}-\mathbf{m}\right|>\varepsilon\right) \rightarrow 0 \quad \text { при } \quad s \rightarrow \infty,
$$

а вероятность

$$
P\left(\left|\boldsymbol{\sigma}^{\prime}-\mathbf{m}\right|<\varepsilon\right) \rightarrow 1 \quad \text { при } \quad s \rightarrow \infty .
$$

Однако последнее означает, что

$$
p^{\prime}\left(\boldsymbol{\sigma}^{\prime}\right) \rightarrow \delta\left(\boldsymbol{\sigma}^{\prime}-\mathbf{m}\right) \quad \text { при } \quad s \rightarrow \infty
$$

Учитывая (6), приходим к равенству (8).

Смысл формулы (8) состоит в том, что оператор Каданова при $s \rightarrow \infty$ nреобразует систему единичных спинов, ориентированных почти хаотически, в систему спинов, равных по модулю $m(\tau)$ и ориентированных в направлении оси $z$. Если бы система, получаемая в результате преобразования Каданова, состояла из единичных спинов, то оператор

$$
\widehat{K}_{\infty}=\lim _{s \rightarrow \infty} \widehat{K}_{s}
$$

можно было бы отождествить с эволюционным оператором $\widehat{\mathcal{E}}_{\tau}$, определенным формулой (2). Однако ввиду того что $m(\tau)<1$ при $\tau<1$, преобразование Каданова нельзя рассматривать как изменение физического состояния системы спинов в процессе эволюции и оно представляет собой лишь некоторое преобразование симметрии.

Понятно, что для того чтобы система, получаемая в результате преобразования, состояла из единичных спинов, действие оператора Каданова нужно дополнить умножением блочных спинов на некоторый коэффициент. 


\section{5. РЕНОРМАЛИЗАЦИОННАЯ ГРУППА}

Преобразование РГ [5] отличается от преобразования Каданова тем, что блочные спины умножаются на коэффициент $s^{|a|}$,

$$
\boldsymbol{\sigma}^{\prime \prime}(s)=s^{|a|} \boldsymbol{\sigma}^{\prime}(s)
$$

Плотность вероятности при этом преобразуется по формуле

$$
p^{\prime \prime}\left(\boldsymbol{\sigma}^{\prime \prime}\right)=\widehat{R}_{s} p(\boldsymbol{\sigma})=\left(\widehat{K}_{s} p(\boldsymbol{\sigma})\right)_{\boldsymbol{\sigma}^{\prime} \rightarrow s^{-|a|}} \boldsymbol{\sigma}^{\prime \prime}
$$

Зафиксируем значение $\tau$ немногим больше нуля и рассмотрим, как действует оператор $\widehat{R}_{s}$ на плотность $p(\tau)$ при $s \rightarrow \infty$.

В силу соотношения (7) и поскольку $s^{|a|} \rightarrow \infty$ при $s \rightarrow \infty$, все перенормированные блочные спины (11) с ростом $s$ ориентируются вдоль оси $z$ и неограниченно растут по модулю,

$$
\boldsymbol{\sigma}^{\prime \prime}(s) \sim s^{|a|} m(\tau) \mathbf{k} \quad \text { при } \quad s \rightarrow \infty,
$$

где

$$
s^{|a|} m(\tau) \rightarrow \infty \quad \text { при } \quad s \rightarrow \infty,
$$

так как $m(\tau) \neq 0$ при $\tau>0$.

Таким образом, предел $\lim _{s \rightarrow \infty} \widehat{R}_{s} p(\tau, \sigma)$ не существует.

Полученный результат можно условно описать следуюшим образом. $B$ nределе $s \rightarrow \infty$ оператор $\widehat{R}_{s}$ преобразует систему единичных спинов, ориентированных почти хаотически, в абсолютно упорядоченную систему спинов, бесконечных по модулю. Последняя система не имеет физического смысла, поэтому оператор РГ, так же как и оператор Каданова, нельзя рассматривать в качестве эволюционного оператора. Он по-прежнему является лишь абстрактным преобразованием симметрии.

Очевидно, что для того чтобы система, получаемая в результате преобразования, состояла из единичных спинов и оператор РГ можно было бы отождествить с эволюционным оператором (2), следует ввести зависимость параметра РГ $s$ от $\tau$. Причем эта зависимость должна быть такой, чтобы рост множителя $s^{|a|}$ в формуле (12) компенсировался убыванием функции $m(\tau)$, т.е. при $s \rightarrow \infty$ необходимо положить

$$
s^{|a|} \sim \frac{1}{m(\tau)} .
$$




\section{6. АСИМПТОТИЧЕСКАЯ ФОРМУЛА ТЕОРИИ РЕНОРМАЛИЗАЦИОННОЙ ГРУППЫ}

Законы критического поведения макроскопических средних (намагниченности, теплоемкости, корреляционной функции $G(0))$ с изменением $\tau$ и соответствуюшие им законы подобия получаются из асимптотической формулы (1) путем введения зависимости параметра РГ $s$ от $\tau$,

$$
s=s(\tau)=c \tau^{-\nu},
$$

где по определению $\nu=1 / y_{1}$. Здесь $y_{1}$ - параметр, входящий в формулу (1). Отметим, что этот шаг означает согласование действия оператора РГ с эволюцией плотности вероятности. В результате формула (1) преобразуется к виду

$$
\widehat{R}_{s(\tau)} \mu(\tau) \approx \mu^{*}+A c^{y_{1}} \mathbf{e}_{1}+f(\tau), \quad \tau \rightarrow 0,
$$

или

$$
\widehat{R}_{s(\tau)} \mu(\tau)=\text { const }+f(\tau), \quad \tau \rightarrow 0,
$$

где $f(\tau)$ - некоторая бесконечно малая функция. Другими словами, сушествует предел

$$
\lim _{\tau \rightarrow 0} \widehat{R}_{s(\tau)} \mu(\tau) .
$$

В терминах плотности распределения это требование означает, что существует предел

$$
\lim _{\tau \rightarrow 0} \widehat{R}_{s(\tau)} p(\tau)
$$

или

$$
\widehat{R}_{s(\tau)} p(\tau)=\mathrm{const}+f(\tau), \quad \tau \rightarrow 0,
$$

где $f(\tau)$ - некоторая бесконечно малая величина. Здесь мы предполагаем, что плотность распределения $p(\tau, \boldsymbol{\sigma})$ является непрерывной функцией параметров $\mu$, как, например, плотность Гинзбурга-Ландау.

Именно формула (16) (или (14)) и позволяет получить все законы критического поведения физических величин в зависимости от $\tau$ и соответствующие законы подобия. Например, намагниченность при действии оператора РГ преобразуется по формуле

$$
m(p(\tau))=s^{a} m\left(\widehat{R}_{s} p(\tau)\right) .
$$

Меняя $s$ по закону (13), получим

$$
m(p(\tau))=\tau^{-\nu a} c^{a} m\left(\widehat{R}_{s(\tau)} p(\tau)\right) .
$$

В силу формулы (16) при малых $\tau$ имеем

$$
m(p(\tau))=\tau^{-\nu a} c^{a} m(\text { const }+f(\tau)), \quad \tau \rightarrow 0,
$$


или

$$
m(p(\tau)) \sim C \tau^{\beta}, \quad \tau \rightarrow 0
$$

где

$$
\beta=-\nu a,
$$

а $C$ - некоторое число. Подчеркнем, что формула (17) ничего не говорит о значении $C$. Ее смысл состоит лишь в том, что $m(p(\tau))=O\left(\tau^{\beta}\right)$ при $\tau \rightarrow 0$.

Исследуем действие оператора $\widehat{R}_{s(\tau)}$ на плотность $p(\tau)$ в пределе малых $\tau$, т.е. найдем

$$
\lim _{\tau \rightarrow 0} \widehat{R}_{s(\tau)} p(\tau)
$$

В результате согласования (13) действия $\widehat{R}_{s}$ с эволюцией плотности как блочные спины, так и множитель $s^{|a|}$ в формуле (11) являются функциями $\tau$, т.е.

$$
\boldsymbol{\sigma}^{\prime \prime}=c^{|a|} \tau^{-\nu|a|} \boldsymbol{\sigma}^{\prime}(\tau)
$$

В силу соотношений (5) и (13) при малых $\tau$ и соответственно при больших $s$ блочный спин совпадает с намагниченностью, поэтому на основании (17) и (6) получим

$$
\boldsymbol{\sigma}^{\prime}(\tau) \sim C \tau^{\beta} \mathbf{k}, \quad \tau \rightarrow 0
$$

и

$$
\boldsymbol{\sigma}^{\prime \prime} \rightarrow \alpha \mathbf{k}, \quad \tau \rightarrow 0
$$

где $\alpha=C c^{|a|}$.

Таким образом,

$$
\lim _{\tau \rightarrow 0} \widehat{R}_{s(\tau)} p(\tau, \boldsymbol{\sigma})=\delta\left(\boldsymbol{\sigma}^{\prime \prime}-\alpha \mathbf{k}\right)=\frac{1}{2 \pi} \delta\left(\sigma^{\prime \prime}-\alpha\right) \delta\left(\sigma_{z}^{\prime \prime}-\sigma^{\prime \prime}\right) .
$$

Последнее означает, что оператор $Р \Gamma \widehat{R}_{s(\tau)}$ в пределе $\tau \rightarrow 0$ преобразует абсолютно хаотическое состояние системы единичных спинов в абсолютно упорядоченное состояние системы спинов, равных по модулю величине $\alpha$.

Подчеркнем, что равенство (19) является необходимым условием для выполнения асимптотической формулы (1) (если, конечно, выполняется естественное предположение о непрерывной зависимости плотности $p$ от параметров $\mu$ ) и необходимылм $u д о-$ статочным условием для выполнения ее следствия (16), порождающего все законы критической зависимости от $\tau$.

Выбирая в (13)

$$
c=C^{-1 /|a|},
$$

получим из (19), что

$$
\lim _{\tau \rightarrow 0} \widehat{R}_{s(\tau)} p(\tau, \boldsymbol{\sigma})=\frac{1}{2 \pi} \delta\left(\boldsymbol{\sigma}^{\prime \prime}-1\right) \delta\left(\sigma_{z}^{\prime \prime}-1\right) .
$$


Отметим, что это равенство, так же как и закон критического поведения (17), можно было бы получить из условия (15) еще проще. Действительно, это условие эквивалентно требованию существования предела

$$
\lim _{\tau \rightarrow 0} \boldsymbol{\sigma}^{\prime \prime}(\tau)=\mathbf{k} c^{|a|} \lim _{\tau \rightarrow 0} \tau^{-\nu|a|} m(\tau)
$$

в соответствии с формулами (18) и (5). Но для этого достаточно, чтобы

$$
m(\tau)=O\left(\tau^{\nu|a|}\right), \quad \tau \rightarrow 0,
$$

т.е. чтобы выполнялось соотношение $(17)$ с некоторым числом $C$. В этом случае

$$
\lim _{\tau \rightarrow 0} \boldsymbol{\sigma}^{\prime \prime}(\tau)=C c^{|a|} \mathbf{k}
$$

Выбрав $c$ в виде (20), получим (21).

Смысл равенства (21) состоит в том, что оператор $P \Gamma \widehat{R}_{s(\tau)}$ в пределе $\tau \rightarrow 0$ nреобразует систему единичных спинов из абсолютно хаотического в абсолютно упорядоченное состояние.

Переобозначив переменные $\boldsymbol{\sigma}^{\prime \prime}$ на $\boldsymbol{\sigma}$, получим из (21), что

$$
\lim _{\tau \rightarrow 0} \widehat{R}_{s(\tau)} p(\tau, \boldsymbol{\sigma})=\frac{1}{2 \pi} \delta(\sigma-1) \delta\left(\sigma_{z}-1\right) .
$$

Сравнивая это равенство с формулой (3), видим, что оператор $P \Gamma \widehat{R}_{\infty}$, определенный соотношением

$$
\widehat{R}_{\infty} p_{0}=\lim _{\tau \rightarrow 0} \widehat{R}_{s(\tau)} p(\tau, \boldsymbol{\sigma})
$$

есть не что иное, как эволючионный оператор $\widehat{\mathcal{E}}_{0}$,

$$
\widehat{R}_{\infty}=\widehat{\mathcal{E}}_{0}
$$

т.е. оператор, упорядочивающий систему спинов.

Из соотношения (22) следует, что

$$
\widehat{R}_{s(\tau)} p(\tau)=\frac{1}{2 \pi} \delta(\sigma-1) \delta\left(\sigma_{z}-1\right)+f(\tau), \quad \tau \rightarrow 0
$$

где $f(\tau)$ - бесконечно малая величина. Поэтому в силу равенства (2) имеем

$$
\widehat{R}_{s(\tau)} p(\tau)=\widehat{\mathcal{E}}_{\tau} p(\tau)+f(\tau), \quad \tau \rightarrow 0
$$

Таким образом, оператор $Р \Gamma$, зависящий от $\tau$ в соответствии с формулой (13), при малых $\tau$ совпадает (в смысле действия на вектор состояния системь спинов) с эволюиионным оператором плотности вероятности (2). 


\section{7. ФИЗИЧЕСКОЕ ОБСУЖДЕНИЕ}

Обсудим связь между принятой трактовкой РГ как преобразования внутри множества моделей данной физической системы и предлагаемой здесь интерпретацией оператора РГ как эволюционного оператора системы.

Оператор РГ меняет параметры модельного гамильтониана $\mu$ и таким образом преобразует один модельный гамильтониан в другой. Поэтому его естественно рассматривать как преобразование внутри множества различных моделей одной физической системы. Однако параметры гамильтониана являются функциями температуры, $\mu=\mu(\tau)$. РГ Каданова-Вильсона является однопараметрической полугруппой. Если ввести определенную зависимость ее параметра от $\tau, s=s(\tau)$ (а такая зависимость всегда вводится при выводе законов критического поведения), то изменение параметров гамильтониана под действием оператора РГ $\mu(s(\tau))$ будет соответствовать изменению температуры. Тогда РГ-преобразование модельного гамильтониана естественно рассматривать уже не как преобразование между различными моделями системы, а как преобразование физического состояния системы с изменением температуры.

Отметим, что полученный результат может быть использован как руководящий принцип или критерий при конструировании модельного гамильтониана физической системы. Действительно, так как соотношение (23), по-сушеству, эквивалентно асимптотической формуле (1), то для того чтобы плотность вероятности, соответствующая конструируемому гамильтониану, порождала законы критического поведения и законы подобия, нужно, чтобы она удовлетворяла уравнению (23).

\section{Список литературы}

[1] Д. В. Ширков. ТМФ. 1984. Т. 60. № 2. С. 218.

[2] В. Ф. Ковалев, Д. В. Ширков. ТМФ. 1999. Т. 121. № 1. С. 66.

[3] D. V. Shirkov, V. F. Kovalev. Phys. Rep. 2001. V. 352. № 4-6. P. 219.

[4] D. O'Connor, C. R. Stephens. Phys. Rep. 2001. V. 352. № 4-6. P. 215.

[5] ШI. Ма. Современная теория критических явлений. М.: Мир, 1980.

[6] К. Вильсон, Джс. Когут. Ренормализационная группа и $\varepsilon$-разложение. М.: Мир, 1975.

[7] В. Ж. Сакбаев. Матем. заметки. 2001. Т. 70. № 3. С. 434.

[8] A. В. Гласко. Мера упорядоченности в статистических системах. Матем. заметки (в печати).

[9] И. Н. Коваленко, А. А. Филиппова. Теория вероятностей и математическая статистика. М.: Высшая школа, 1982. 